\title{
RAMANUJAN-TYPE IDENTITIES FOR SHIMURA CURVES
}

\author{
YIFAN YANG
}

\begin{abstract}
In 1914, Ramanujan gave a list of 17 identities expressing $1 / \pi$ as linear combinations of values of hypergeometric functions at certain rational numbers. Since then, identities of similar nature have been discovered by many authors. Nowadays, one of the standard approaches to this kind of identities uses the theory of modular curves. In this paper, we will consider the case of Shimura curves and obtain Ramanujan-type formulas involving special values of hypergeometric functions and products of Gamma values. These products of Gamma values are related to periods of elliptic curves with complex multiplication by $\mathbb{Q}(\sqrt{-3})$ and $\mathbb{Q}(\sqrt{-4})$.
\end{abstract}

\section{INTRODUCTION}

Among all the amazing formulas discovered by Ramanujan, the identity

$$
\sum_{n=0}^{\infty} \frac{(6 n+1)(1 / 2)_{n}^{3}}{(n !)^{3}}\left(\frac{1}{4}\right)^{n}=\frac{4}{\pi}
$$

involving hypergeometric functions and $\pi$ is perhaps one of the most well-known, where $(a)_{n}$ is the Pochhammer symbol $(a)_{n}=a(a+1) \ldots(a+n-1)$. In [14], he gave 17 such identities. Since then, number theorists [4, 5, 6, 7, 8, 9, 10, 11, 19] have produced many more identities of similar nature. (See [3] for a comprehensive survey on the history of Ramanujan-type series for $1 / \pi$.) Nowadays, it is well-understood that Ramanujan's $1 / \pi$-series are related to the CM-theory and the Hecke theory of modular curves. Since Shimura curves are generalizations of modular curves and there are analogues of CM points and Hecke operators in the setting of Shimura curves, one may wonder whether we have also Ramanujan-type formulas for Shimura curves. In this paper, we consider the AtkinLehner quotient $X_{6}^{*}$ of the Shimura curve associated to a maximal order in the quaternion algebra of discriminant 6 over $\mathbb{Q}$ by all the Atkin-Lehner involutions. Using the method developed in our earlier work [18] for computing Hecke operators relative to the explicit bases of automorphic forms in terms of solutions of Schwarzian differential equations, we obtain Ramanujan-type identities for $X_{6}^{*}$.

To state the results, let us recall the Chowla-Selberg formula [15], which states that if $E$ is an elliptic curve over $\overline{\mathbb{Q}}$ with complex multiplication by an imaginary quadratic number field $K$ of discriminant $d$, then, up to an algebraic factor, the period of $E$ is

$$
\Omega_{d}=\sqrt{\pi} \prod_{0<a<|d|} \Gamma\left(\frac{a}{|d|}\right)^{w_{d} \chi_{d}(a) / 4 h_{d}},
$$

Date: October 7, 2018.

2000 Mathematics Subject Classification. Primary 11F12 secondary 11Y60.

The author was partially supported by Grant 99-2115-M-009-011-MY3 of the National Science Council, Taiwan (R.O.C.). 
where $\chi_{d}$ is the Kronecker character associated to $K, w_{d}$ is the number of roots of unity in $K$, and $h_{d}$ is the class number of $K$. Then we have the following Ramanujan-type identities for the Shimura curve $X_{6}^{*}$.

Theorem 1. Let

$$
\begin{array}{ll}
A_{n}=\frac{(1 / 12)_{n}(1 / 4)_{n}(5 / 12)_{n}}{(1 / 2)_{n}(3 / 4)_{n} n !}, & A_{n}^{\prime}=\frac{(7 / 12)_{n}(3 / 4)_{n}(11 / 12)_{n}}{(3 / 2)_{n}(5 / 4)_{n} n !} \\
B_{n}=\frac{(1 / 12)_{n}(1 / 3)_{n}(7 / 12)_{n}}{(2 / 3)_{n}(5 / 6)_{n} n !}, & B_{n}^{\prime}=\frac{(5 / 12)_{n}(2 / 3)_{n}(11 / 12)_{n}}{(4 / 3)_{n}(7 / 6)_{n} n !}
\end{array}
$$

and

$$
C_{1}=\frac{4}{\sqrt[4]{12}} \frac{\pi}{\Omega_{-4}^{2}}=\frac{4}{\sqrt[4]{12}} \frac{\Gamma(3 / 4)^{2}}{\Gamma(1 / 4)^{2}}, \quad C_{2}=\frac{3}{\sqrt[6]{2}} \frac{\pi}{\Omega_{-3}^{2}}=\frac{3}{\sqrt[6]{2}} \frac{\Gamma(2 / 3)^{3}}{\Gamma(1 / 3)^{3}}
$$

Then

$$
\begin{gathered}
\sum_{n=0}^{\infty}\left(R_{1} n+R_{2}\right) A_{n}\left(\frac{M}{N}\right)^{n}=R_{3}^{1 / 2}|M|^{3 / 4} N^{1 / 4} C_{1} \\
\sum_{n=0}^{\infty}\left(R_{1} n+R_{1} / 2+R_{2}\right) A_{n}^{\prime}\left(\frac{M}{N}\right)^{n}=R_{3}^{1 / 2}|M|^{1 / 4} N^{3 / 4} C_{1}^{-1}
\end{gathered}
$$

hold for the following values of $M, N, R_{1}, R_{2}$ and $R_{3}$.

\begin{tabular}{r|rrrrr}
\hline \hline$D$ & $M$ & $N$ & $R_{1}$ & $R_{2}$ & $R_{3}$ \\
\hline-120 & $-7^{4}$ & $3^{3} \cdot 5^{3}$ & 74480 & $6860 / 3$ & 5 \\
-52 & $2^{2} \cdot 3^{7}$ & $5^{6}$ & 64584 & 972 & 1 \\
-132 & $2^{4} \cdot 11^{2}$ & $5^{6}$ & 226512 & 1936 & 11 \\
-75 & $-11^{4}$ & $2^{10} \cdot 3^{3} \cdot 5$ & 946220 & $33275 / 3$ & 2 \\
-43 & $-3^{7} \cdot 7^{4}$ & $2^{10} \cdot 5^{6}$ & 159971868 & 3417309 & 2 \\
-88 & $3^{7} \cdot 7^{4}$ & $5^{6} \cdot 11^{3}$ & 324590112 & 3667356 & 11 \\
-312 & $7^{4} \cdot 23^{4}$ & $5^{6} \cdot 11^{6}$ & 212826755232 & 901428696 & 6 \\
-148 & $2^{2} \cdot 3^{7} \cdot 7^{4} \cdot 11^{4}$ & $5^{6} \cdot 17^{6}$ & 1027794972204 & 17528128002 & 1 \\
\hline \hline
\end{tabular}

Also,

$$
\begin{gathered}
\sum_{n=0}^{\infty}\left(R_{1} n+R_{2}\right) B_{n}\left(\frac{M}{N}\right)^{n}=R_{3}^{1 / 2}|M|^{2 / 3} N^{1 / 3} C_{2} \\
\sum_{n=0}^{\infty}\left(R_{1} n+R_{1} / 3+R_{2}\right) B_{n}^{\prime}\left(\frac{M}{N}\right)^{n}=R_{3}^{1 / 2}|M|^{1 / 3} N^{2 / 3} C_{2}^{-1}
\end{gathered}
$$


hold for the following values of $M, N, R_{1}, R_{2}$, and $R_{3}$.

\begin{tabular}{r|rrrrr}
\hline \hline$D$ & $M$ & $N$ & $R_{1}$ & $R_{2}$ & $R_{3}$ \\
\hline-84 & $3^{3}$ & $2^{2} \cdot 7^{2}$ & 4914 & $189 / 2$ & 42 \\
-40 & $-5^{3}$ & $3^{7}$ & 10200 & 175 & 2 \\
-51 & $2^{10}$ & $7^{4}$ & 14688 & 384 & 2 \\
-19 & $-2^{10}$ & $3^{7}$ & 23712 & 896 & 2 \\
-168 & $5^{6}$ & $7^{2} \cdot 11^{4}$ & 10773000 & 118125 & 42 \\
-228 & $-3^{6} \cdot 5^{6}$ & $2^{6} \cdot 7^{4} \cdot 19^{2}$ & 3146100750 & $176023125 / 2$ & 114 \\
-123 & $2^{10} \cdot 5^{6}$ & $7^{4} \cdot 19^{4}$ & 1323972000 & 18960000 & 2 \\
-100 & $-11^{6}$ & $2^{4} \cdot 3^{7} \cdot 5 \cdot 7^{4}$ & 820122270 & $10907545 / 2$ & 2 \\
-147 & $2^{10} \cdot 3^{3} \cdot 5^{6} \cdot 7$ & $11^{4} \cdot 23^{4}$ & 86680314000 & 2593080000 & 42 \\
-67 & $-2^{16} \cdot 5^{6}$ & $3^{7} \cdot 7^{4} \cdot 11^{4}$ & 219324768000 & 2193920000 & 2 \\
-372 & $3^{3} \cdot 5^{6} \cdot 11^{6}$ & $2^{2} \cdot 7^{4} \cdot 19^{4} \cdot 31^{2}$ & 62650568301750 & $3597211344375 / 2$ & 186 \\
-408 & $3^{6} \cdot 5^{6} \cdot 17^{3}$ & $7^{4} \cdot 11^{4} \cdot 31^{4}$ & 33316016526000 & 159415290000 & 1 \\
\hline \hline
\end{tabular}

(Note that the entries in the first columns of the two tables indicate the discriminants of the corresponding CM-points.)

Note that the power series $\sum_{n} A_{n} x^{n}$ is in fact

$$
\sum_{n=0}^{\infty} A_{n} x^{n}={ }_{3} F_{2}\left(\frac{1}{12}, \frac{1}{4}, \frac{5}{12} ; \frac{1}{2}, \frac{3}{4} ; x\right)={ }_{2} F_{1}\left(\frac{1}{24}, \frac{5}{24} ; \frac{3}{4} ; x\right)^{2} .
$$

The hypergeometric differential equation associated to ${ }_{2} F_{1}(1 / 24,5 / 24 ; 3 / 4 ; x)$ is essentially the Schwarzian differential equation associated to the Hauptmodul of $X_{6}^{*}$ that takes values 0,1 , and $\infty$ at the CM-points of discriminants $-4,-24$, and -3 , respectively. Likewise, the power series $\sum_{n} B_{n} x^{n}$ is the square of a ${ }_{2} F_{1}$-hypergeometric function whose corresponding hypergeometric differential equation is essentially the Schwarzian differential equation associated to the Hauptmodul of $X_{6}^{*}$ that has values 0,1 , and $\infty$ at the CM-points of discriminants $-3,-24$, and -4 , respectively. Thus, if we let $\Omega_{\infty}=\infty$ be the period of a generalized elliptic curve (which is natural in view of the well-known fact that the period of the elliptic curve $y^{2}=x(x-1)(x-\lambda)$ is $\pi_{2} F_{1}(1 / 2,1 / 2 ; 1 ; \lambda)$ ), the general form of the Ramanujan's identities and our identities is

$$
\sum_{n=0}^{\infty}\left(R_{1} n+R_{2}\right) C_{n} x_{0}^{n}=\frac{R_{3} \pi}{\Omega_{d}^{2}},
$$

where $R_{1}, R_{2}, R_{3} \in \overline{\mathbb{Q}}, \sum_{n} C_{n} x^{n}$ is the power series expansion of a meromorphic modular form of weight 2 with respect to a Hauptmodul $x$ of a modular curve or a Shimura curve such that $x$ takes value 0 at a CM-point of discriminant $d$ (possibly $\infty$ ), and $x_{0}$ is the value of $x$ at some CM-point of discriminant $d^{\prime} \neq d$.

Remark 1. There are three more pairs $(M, N)$ of integers such that the first set of equalities hold for some $R_{1}, R_{2}$, and $R_{3}$. However, a rigorous proof of these three cases will require the computation of Hecke operators $T_{p}$ on the spaces of automorphic forms of weight $8(p+1)$, for $p=29,31$, and 41 , respectively. Such a computation will take a 
considerable amount of time and computer resource. Numerically, we find that for

\begin{tabular}{r|rr}
\hline \hline$D$ & \multicolumn{2}{|c}{$M$} \\
\hline-232 & $-3^{7} \cdot 7^{4} \cdot 11^{4} \cdot 19^{4}$ & $5^{6} \cdot 23^{6} \cdot 29^{3}$ \\
-708 & $2^{8} \cdot 7^{4} \cdot 11^{4} \cdot 47^{4} \cdot 59^{2}$ & $5^{6} \cdot 17^{6} \cdot 29^{6}$ \\
-163 & $-3^{11} \cdot 7^{4} \cdot 19^{4} \cdot 23^{4}$ & $2^{10} \cdot 5^{6} \cdot 11^{6} \cdot 17^{6}$ \\
\hline \hline
\end{tabular}

the first set of identities hold with

\begin{tabular}{r|rrr}
\hline \hline$D$ & $R_{1}$ & $R_{2}$ & $R_{3}$ \\
\hline-232 & 2443514820645762864 & 37910578302227862 & 58 \\
-708 & 6167093737665689901072 & 99165724038088434496 & 59 \\
-163 & 3071651418339975941652 & 15473113111724338791 & 2 \\
\hline \hline
\end{tabular}

Likewise, numerically, we find that the second set of identities holds with $M=2^{16} \cdot 5^{6} \cdot 11^{6}$, $N=7^{4} \cdot 31^{4} \cdot 43^{4}, R_{1}=45769617921456000, R_{2}=1027334373120000$, and $R_{3}=2$. This corresponds to the CM-point of discriminant -267 . A rigorous proof of this case requires the computation of $T_{23}$ on the space of automorphic forms of weight 192 on $X_{6}^{*}$.

Note that if we allow $M / N$ to be irrational algebraic numbers, then there are infinitely many Ramanujan-type identities.

Remark 2. Observe that when a prime $p \neq 2,3$ divides $M$, the series in the theorem converges $p$-adically. One may wonder what kind of $p$-adic numbers they converge to. Quite interestingly and mysteriously, it appears that with $C_{1}$ and $C_{2}$ replaced by certain products of $p$-adic Gamma values and possibly $R_{3}$ by different rational numbers, the identities also hold $p$-adically.

Let $\Gamma_{p}(x)$ be the $p$-adic Gamma function defined by

$$
\Gamma_{p}(n)=(-1)^{n} \prod_{0<j<n, p \nmid j} j
$$

for positive integers $n$ and extended continuously to $\mathbb{Z}_{p}$. Let

$$
C_{p}=\frac{3^{6}}{2} \frac{\Gamma_{p}(2 / 3)^{9}}{\Gamma_{p}(1 / 3)^{9}}
$$

Our numerical computation suggests that, for the second set of identities in Theorem 1 . when $p \neq 2,3$ divides $M$,

$$
\begin{aligned}
\sum_{n=0}^{\infty}\left(R_{1} n+R_{2}\right) B_{n}\left(\frac{M}{N}\right)^{n} & =\sqrt[6]{R_{3}^{3} M^{4} N^{2} C_{p}} \\
\sum_{n=0}^{\infty}\left(R_{1} n+R_{1} / 3+R_{2}\right) B_{n}^{\prime}\left(\frac{M}{N}\right)^{n} & =p \sqrt[6]{R_{3}^{3} M^{2} N^{4} / C_{p}}
\end{aligned}
$$

hold $p$-adically with the same $R_{1}, R_{2}$, but possibly different $R_{3}$. For example, for $p=5$, the equality holds with

\begin{tabular}{c|ccccccccc}
\hline \hline$D$ & -40 & -168 & -228 & -123 & -147 & -67 & -372 & -408 & -267 \\
\hline$R_{3}$ & 2 & 42 & -38 & 2 & $21 / 2$ & 2 & -62 & -3 & $1 / 2$ \\
\hline \hline
\end{tabular}

(There is a unique sixth root such that the equality holds.) The equalities are verified up to 1005 -adic digits. There also appears to be $p$-adic analogues for the first set of identities in 
Theorem 1 in which the limits are $\Gamma_{p}(3 / 4) / \Gamma_{p}(1 / 4)$ times some algebraic numbers. We believe that the identities should be related to $p$-adic periods of elliptic curves with $\mathrm{CM}$, but we do not have a proof of these identities yet.

The plan for the rest of the paper is as follow. In Sections 2.1 2.4 we set up our notations and review basic properties of the Shimura curve under consideration. In particular, in Section 2.2, we will give an explicit description of automorphic forms on the Shimura curve in terms of hypergeometric functions. The computation of Hecke operators relative to these automorphic forms is pivotal in the proof of the Ramanujan-type identities. In Section 2.5. we state several general identities, the specialization of which gives us the identities in Theorem 1. The proof of these general identities will be given in Section 3 In Section 4, we prove the identities in Theorem 1. The main task there is the evaluation of certain automorphic functions on Shimura curves associated to Eichler orders of the quaternion algebra of discriminant 6 over $\mathbb{Q}$ at CM-points. The evaluation relies on some auxiliary polynomials, which will be listed in the appendix.

\section{RAMANUJAN-TYPE FORMULAS FOR SHIMURA CURVES}

2.1. The Shimura curve $X_{6}^{*}$. Let the quaternion algebra of discriminant 6 over $\mathbb{Q}$ be presented by $B=\left(\frac{-1,3}{\mathbb{Q}}\right)$, i.e., the algebra generated by $I$ and $J$ over $\mathbb{Q}$ with the relations

$$
I^{2}=-1, \quad J^{2}=3, \quad I J=-J I .
$$

Choose the embedding $\iota: B \rightarrow M(2, \mathbb{R})$ to be

$$
I \longmapsto\left(\begin{array}{cc}
0 & -1 \\
1 & 0
\end{array}\right), \quad J \longmapsto\left(\begin{array}{cc}
\sqrt{3} & 0 \\
0 & -\sqrt{3}
\end{array}\right)
$$

as in [1, Section 5.5.2]. Fix the maximal order $\mathcal{O}$ to be $\mathbb{Z}+\mathbb{Z} I+\mathbb{Z} J+\mathbb{Z}(1+I+J+I J) / 2$. Then the image of the norm-one group of $\mathcal{O}$ under the embedding $\iota$ is

$$
\Gamma=\left\{\frac{1}{2}\left(\begin{array}{cc}
\alpha & \beta \\
-\beta^{\prime} & \alpha^{\prime}
\end{array}\right) \in \operatorname{SL}(2, \mathbb{R}): \alpha, \beta \in \mathbb{Z}[\sqrt{3}], \alpha \equiv \beta \bmod 2\right\},
$$

where $\alpha^{\prime}$ and $\beta^{\prime}$ denote the Galois conjugates of $\alpha$ and $\beta$, respectively. Let also

$$
\Gamma^{*}=\left\{\frac{1}{\mathrm{~N}(\gamma)^{1 / 2}} \iota(\gamma): \gamma \in N_{B}(\mathcal{O}), \mathrm{N}(\gamma)>0\right\}=\gamma_{1} \Gamma \cup \gamma_{2} \Gamma \cup \gamma_{3} \Gamma \cup \gamma_{6} \Gamma,
$$

where $N_{B}(\mathcal{O})$ is the normalizer of $\mathcal{O}$ in $B$, and

$$
\begin{aligned}
& \gamma_{1}=\iota(1)=\left(\begin{array}{ll}
1 & 0 \\
0 & 1
\end{array}\right), \quad \gamma_{2}=\frac{1}{\sqrt{2}} \iota(1+I)=\frac{1}{\sqrt{2}}\left(\begin{array}{cc}
1 & -1 \\
1 & 1
\end{array}\right), \\
& \gamma_{3}=\frac{1}{\sqrt{3}} \iota((3+3 I+J+K) / 2)=\frac{1}{2 \sqrt{3}}\left(\begin{array}{cc}
3+\sqrt{3} & -3+\sqrt{3} \\
3+\sqrt{3} & 3-\sqrt{3}
\end{array}\right), \\
& \gamma_{6}=\frac{1}{\sqrt{6}} \iota(3 I+J)=\left(\begin{array}{cc}
0 & -3+\sqrt{3} \\
3+\sqrt{3} & 0
\end{array}\right) .
\end{aligned}
$$

Let $X_{6}=\Gamma \backslash \mathbb{H}$ and $X_{6}^{*}=\Gamma^{*} \backslash \mathbb{H}$ be the Shimura curves associated to $\mathcal{O}$ and $N_{B}(\mathcal{O})$, respectively. In particular, $X_{6}^{*}=X_{6} / W_{6}$ is the quotient of $X_{6}$ by all the Atkin-Lehner involutions. A fundamental domain for $X_{6}^{*}$ is given by 


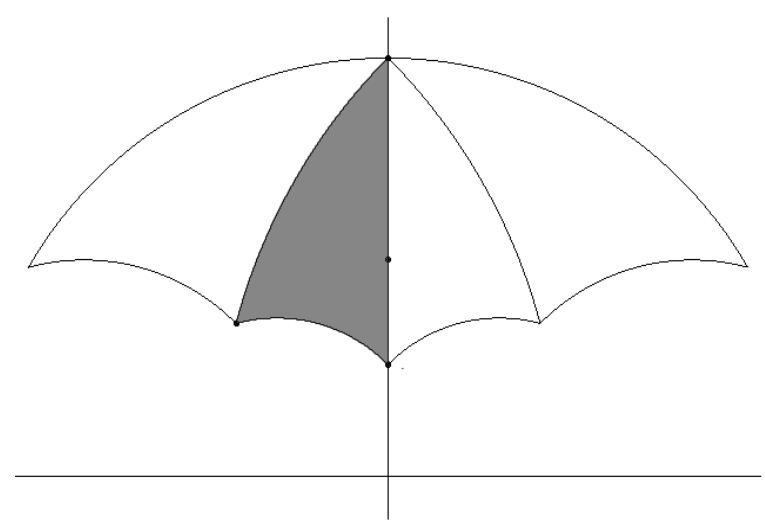

(See [1, Figure 5.1] and [17].) Here the grey area represents a fundamental domain for $X_{6}^{*}$. The grey area, together with the three white areas, forms a fundamental domain for $X_{6}$. The four marked points on the boundary of the grey area are

$$
P_{4}=i, \quad P_{6}=\frac{-1+i}{1+\sqrt{3}}, \quad(2-\sqrt{3}) i, \quad P_{2}=\frac{(\sqrt{6}-\sqrt{2}) i}{2},
$$

respectively. The points $P_{2}, P_{4}$, and $P_{6}$ are representatives of the elliptic points of orders 2 , 4 , and 6 , respectively. They are CM-points of discriminants $-24,-4$, and -3 , respectively. Their isotropy subgroups are generated by

$$
M_{2}=\frac{1}{\sqrt{6}}\left(\begin{array}{cc}
0 & -3+\sqrt{3} \\
3+\sqrt{3} & 0
\end{array}\right), \quad M_{4}=\frac{1}{\sqrt{2}}\left(\begin{array}{cc}
1 & -1 \\
1 & 1
\end{array}\right)
$$

and

$$
M_{6}=\frac{1}{2 \sqrt{3}}\left(\begin{array}{cc}
3+\sqrt{3} & 3-\sqrt{3} \\
-3-\sqrt{3} & 3-\sqrt{3}
\end{array}\right),
$$

respectively. The point $(2-\sqrt{3}) i$ is equivalent to $P_{4}$ through $M_{2}$.

2.2. Spaces of automorphic forms on $X_{6}^{*}$. In [18], we showed that when a Shimura curve has genus 0 , all automorphic forms can be expressed in terms of solutions of its Schwarzian differential equations. When a Shimura curve corresponds a triangle group, this Schwarzian differential equation is essentially a hypergeometric differential equation. In this section, we review the properties relevant to our consideration.

Lemma 3. Let $t(\tau)$ be the Hauptmodul of $X_{6}^{*}$ that takes values 0,1 , and $\infty$ at the elliptic points of order 4, 2, and 6, respectively. Let

$$
F_{1}(t)={ }_{2} F_{1}\left(\frac{1}{24}, \frac{5}{24} ; \frac{3}{4} ; t\right), \quad F_{2}(t)=t^{1 / 4}{ }_{2} F_{1}\left(\frac{7}{24}, \frac{11}{24} ; \frac{5}{4} ; t\right),
$$

be two linearly independent solutions of

$$
\theta\left(\theta-\frac{1}{4}\right)-t\left(\theta+\frac{1}{24}\right)\left(\theta+\frac{5}{24}\right)=0, \quad \theta=t \frac{d}{d t} .
$$

Then we have

$$
C \frac{F_{2}(t)}{F_{1}(t)}=\frac{\tau-P_{4}}{\tau-\bar{P}_{4}}=\frac{\tau-i}{\tau+i}
$$


where

$$
\begin{aligned}
C & =\frac{P_{2}-P_{4}}{P_{2}-\bar{P}_{4}} \frac{\Gamma(3 / 4) \Gamma(19 / 24) \Gamma(23 / 24)}{\Gamma(5 / 4) \Gamma(13 / 24) \Gamma(17 / 24)} \\
& =(\sqrt{2}-\sqrt{3}) \frac{\Gamma(3 / 4) \Gamma(19 / 24) \Gamma(23 / 24)}{\Gamma(5 / 4) \Gamma(13 / 24) \Gamma(17 / 24)}=-\frac{4}{\sqrt[4]{12}} \frac{\Gamma(3 / 4)^{2}}{\Gamma(1 / 4)^{2}} .
\end{aligned}
$$

Moreover, we have

(5) $t^{\prime}(\tau)=\frac{4 t^{3 / 4}(1-t)^{1 / 2}}{C\left(P_{4}-\bar{P}_{4}\right)}\left(F_{1}(t)-C F_{2}(t)\right)^{2}=\frac{2 t^{3 / 4}(1-t)^{1 / 2}}{C i}\left(F_{1}(t)-C F_{2}(t)\right)^{2}$,

valid for $\tau$ in the fundamental domain such that $|t(\tau)|<1$. Here $t(\tau)^{1 / 4}$ is defined in a way such that it becomes a holomorphic function near $P_{4}$ and takes positive real values along the boundary of the fundamental domain from $P_{4}$ to $P_{2}$. Likewise $(1-t(\tau))^{1 / 2}$ is defined in a way such that it is a holomorphic function near $P_{2}$ and takes positive values along the boundary from $P_{2}$ and $P_{6}$.

Proof. The proof is similar to that of Lemma 14 in [18], so we omit it. Here we just point out that the simplification of the constant $C$ uses the Gauss multiplication formula

$$
\Gamma(z) \Gamma(z+1 / k) \ldots \Gamma(z+(k-1) / k)=(2 \pi)^{(k-1) / 2} k^{1 / 2-k z} \Gamma(k z)
$$

and the functional equation

$$
\Gamma(z) \Gamma(1-z)=\frac{\pi}{\sin (\pi z)}
$$

so that

$$
\begin{aligned}
\left(\frac{c_{19} c_{23}}{c_{13} c_{17}}\right)^{2} & =\frac{c_{3} c_{7} c_{11} c_{15} c_{19} c_{23}}{c_{1} c_{5} c_{9} c_{13} c_{17} c_{21}} \cdot \frac{c_{9} c_{21}}{c_{3} c_{15}} \cdot \frac{c_{1} c_{23}}{c_{7} c_{17}} \cdot \frac{c_{5} c_{19}}{c_{11} c_{13}} \\
& =6^{-1 / 2} \frac{\Gamma(3 / 4)}{\Gamma(1 / 4)} \cdot 2^{-1 / 2} \frac{\Gamma(3 / 4)}{\Gamma(1 / 4)} \cdot \frac{\sin (\pi / 24)}{\sin (7 \pi / 24)} \cdot \frac{\sin (5 \pi / 24)}{\sin (11 \pi / 24)} \\
& =\frac{(\sqrt{3}-\sqrt{2})^{2}}{\sqrt{12}} \frac{\Gamma(3 / 4)^{2}}{\Gamma(1 / 4)^{2}}
\end{aligned}
$$

where $c_{j}=\Gamma(j / 24)$.

Lemma 4 ([18, Theorem 9]). Let $t(\tau)$ be the Hauptmodul of $X_{6}^{*}$ that takes values 0,1 , and $\infty$ at the elliptic points of order 4, 2, and 6 , respectively. For a positive even integer $k$, let

$$
d_{k}=1-k+\left\lfloor\frac{k}{4}\right\rfloor+\left\lfloor\frac{3 k}{8}\right\rfloor+\left\lfloor\frac{5 k}{12}\right\rfloor
$$

be the dimension of the space $S_{k}\left(X_{6}^{*}\right)$ of automorphic forms of weight $k$ on $X_{6}^{*}$. Then a basis for $S_{k}\left(X_{6}^{*}\right)$ is

$$
t^{j} t^{\{3 k / 8\}}(1-t)^{\{k / 4\}}\left(F_{1}(t)-C F_{2}(t)\right)^{k}, \quad j=0, \ldots, d_{k}-1 .
$$

2.3. CM-points on $X_{6}^{*}$. For a negative integer $d$ with $d \equiv 0,1 \bmod 4$, we let $R_{d}$ denote the imaginary quadratic order of discriminant $d$ and $K_{d}$ be the field of fractions of $R_{d}$. Recall that $K_{d}$ can be imbedded in $B$ if and only if neither 2 nor 3 splits in $K_{d}$. An optimal embedding of $K_{d}$ into $B$ relative to $\left(\mathcal{O}, R_{d}\right)$ is an embedding $\phi: K_{d} \hookrightarrow B$ such that $\phi\left(K_{d}\right) \cap \mathcal{O}=\phi\left(R_{d}\right)$. We let $\Lambda(\mathcal{O}, d)$ denote the set of all optimal embeddings relative to 
$\left(\mathcal{O}, R_{d}\right)$. Note that for each $\phi \in \Lambda(\mathcal{O}, d)$, there is a unique point $\tau_{\phi}$ in the upper half-plane such that

$$
\iota(\phi(a)) \tau_{\phi}=\tau_{\phi}
$$

for all $a \in K_{d}^{\times}$. We call $\tau_{\phi}$ the CM-point of discriminant $d$ associated to $\phi$. (In terms of moduli spaces, the endomorphism ring of the corresponding abelian surface has center $R_{d}$.)

Now it is clear that if $\phi \in \Lambda(\mathcal{O}, d)$, then so is $\gamma^{-1} \phi \gamma$ for any $\gamma \in N_{B}(\mathcal{O})$. In fact, $\Lambda(\mathcal{O}, d) / N_{B}(\mathcal{O})$ is a finite set. In the case when $d$ is a fundamental discriminant, the cardinality is given by the formula

$$
\left|\Lambda(\mathcal{O}, d) / N_{B}(\mathcal{O})\right|= \begin{cases}1, & \text { if } d=-3,-4,-24, \\ \frac{h(d)}{4}\left(1-\left(\frac{d}{2}\right)\right)\left(1-\left(\frac{d}{3}\right)\right), & \text { else, }\end{cases}
$$

where $h(d)$ denotes the class number of $R_{d}$. (See [16].) From now on, we assume that the representatives of $\Lambda(\mathcal{O}, d) / N_{B}(\mathcal{O})$ are chosen such that their corresponding CM-points are inside the fundamental domain drawn above. Moreover, if $\tau$ is a CM-point of discriminant $d$ (fundamental or not) in the fundamental domain with the corresponding optimal embedding $\phi_{\tau}$, we let $\alpha_{\tau}$ denote

$$
\alpha_{\tau}=\phi_{\tau}(\sqrt{d}) .
$$

Lemma 5. Let $t$ be the Hauptmodul of $X_{6}^{*}$ that takes values 0,1 , and $\infty$ at the elliptic points of orders 4, 2, and 6, respectively. Suppose that $\tau$ is a CM-point of discriminant $d$ in the fundamental domain and write $\alpha_{\tau}$ as $a_{0}+a_{1} I+a_{2} J+a_{3} I J$. If $t(\tau)$ takes a value in the line segment $[0,1]$, then $a_{0}=a_{2}=0$. If $t(\tau)$ takes a value in $[1, \infty)$, then $a_{1}=3 a_{3}$. If $t(\tau)$ takes a negative value, then $a_{0}=0$ and $a_{2}=-a_{3}$.

Proof. It is clear that $\operatorname{tr}\left(\alpha_{d}\right)=0$. Thus, $a_{0}=0$. Also, the fixed point of $\iota\left(a_{1} I+a_{2} J+\right.$ $\left.a_{3} I J\right)$ in the upper half-plane is

$$
\frac{a_{2} \sqrt{3}+\epsilon \sqrt{d}}{a_{1}+a_{3} \sqrt{3}},
$$

where $\epsilon=\operatorname{sgn}\left(a_{1}+a_{3} \sqrt{3}\right)$. Now if $t(\tau)$ takes a value in [0,1], then $\tau$ lies on the vertical line segment from $P_{4}$ to $P_{2}$. Thus, $\operatorname{Re} \tau=0$ and we must have $a_{2}=0$.

If $t(\tau)$ takes a value in $[1, \infty)$, then $\tau$ lies on the arc from $P_{6}$ to $P_{2}$, which is part of the circle of $|\tau|^{2}=2-\sqrt{3}$. Thus,

$$
\frac{3 a_{2}^{2}}{\left(a_{1}+a_{3} \sqrt{3}\right)^{2}}+\frac{a_{1}^{2}-3 a_{2}^{2}-3 a_{3}^{2}}{\left(a_{1}+a_{3} \sqrt{3}\right)^{2}}=2-\sqrt{3},
$$

which yields

$$
\left(a_{1}-3 a_{3}\right)^{2}-\left(a_{1}-3 a_{3}\right)\left(a_{1}-a_{3}\right) \sqrt{3}=0 .
$$

Therefore, $a_{1}=3 a_{3}$.

If $t(\tau)$ takes a negative value, then $\tau$ lies on the arc from $P_{4}$ to $P_{6}$, which is part of the circle $(\operatorname{Re} \tau-1)^{2}+(\operatorname{Im} \tau)^{2}=2$. Therefore,

$$
\left(\frac{a_{2} \sqrt{3}}{a_{1}+a_{3} \sqrt{3}}-1\right)^{2}+\frac{a_{1}^{2}-3 a_{2}^{2}-3 a_{3}^{2}}{\left(a_{1}+a_{3} \sqrt{3}\right)^{2}}=2 .
$$

Simplifying, we find $a_{2}=-a_{3}$. 
2.4. Hecke operators on the spaces of automorphic forms on $X_{6}^{*}$. To define the Hecke operator $T_{p}$ on $S_{k}\left(X_{6}^{*}\right)$ for a prime $p$ relatively prime to 6 , we pick an element $\alpha$ in $\mathcal{O}$ of norm $p$ and consider the double coset

$$
\Gamma_{p}^{*}=\Gamma^{*} \iota(\alpha) \Gamma^{*}=\gamma_{1} \Gamma_{p} \cup \gamma_{2} \Gamma_{p} \cup \gamma_{3} \Gamma_{p} \cup \gamma_{6} \Gamma_{p},
$$

where $\gamma_{1}, \gamma_{2}, \gamma_{3}, \gamma_{6}$ are defined by [2], and

$$
\Gamma_{p}=\{\alpha \in \mathcal{O}: \mathrm{N}(\alpha)=p\} .
$$

Here $\mathrm{N}(\alpha)$ denotes the reduced norm of $\alpha$. The group $\Gamma^{*}$ acts on the left of $\Gamma_{p}^{*}$ by multiplication and the number of orbits is $p+1$.

For an automorphic form $G(\tau)$ of weight $k$ in $S_{k}\left(X_{6}^{*}\right)$ and an element $\gamma=\left(\begin{array}{ll}a & b \\ c & d\end{array}\right) \in \Gamma_{p}^{*}$, we define the slash operator by

$$
\left.G(\tau)\right|_{k} \gamma=\frac{(\operatorname{det} \gamma)^{k / 2}}{(c \tau+d)^{k}} G\left(\frac{a \tau+b}{c \tau+d}\right) .
$$

It is easy to show that if $\gamma_{1}$ and $\gamma_{2}$ is in the same coset $\Gamma^{*} \backslash \Gamma_{p}^{*}$, then $\left.G\right|_{k} \gamma_{1}=\left.G\right|_{k} \gamma_{2}$ and the function

$$
\left.\sum_{\gamma \in \Gamma^{*} \backslash \Gamma_{p}^{*}} G(\tau)\right|_{k} \gamma
$$

is again an automorphic form of weight $k$ on $X_{6}^{*}$. In other words, the mapping

$$
T_{p}:\left.G(\tau) \longmapsto p^{k / 2-1} \sum_{\gamma \in \Gamma^{*} \backslash \Gamma_{p}^{*}} G(\tau)\right|_{k} \gamma
$$

defines a linear transformation on $S_{k}\left(X_{6}^{*}\right)$. This linear transformation is called the $p$ th Hecke operator of $S_{k}\left(X_{6}^{*}\right)$. For general integers $n$ relatively prime to 6 , the $n$th Hecke operator $T_{n}$ is defined similarly, with $\Gamma_{p}^{*}$ replaced by $\Gamma_{n}^{*}=\Gamma_{n} \cup \gamma_{2} \Gamma_{n} \cup \gamma_{3} \Gamma_{n} \cup \gamma_{6} \Gamma_{n}$, where $\Gamma_{n}=\{\alpha \in \mathcal{O}: \mathrm{N}(\alpha)=n\}$.

Note that for an element $\gamma \in \gamma_{e} \Gamma_{n}, e \in\{1,2,3,6\}$, the trace of $\sqrt{e} \gamma$ is an integer multiple of $e$. Let $t=\operatorname{tr}(\sqrt{e} \gamma) / e$. If $t$ satisfies $(e t)^{2}<4 e n$, then the fixed point of $\gamma$ is a CM-point of discriminant $\left(e^{2} t^{2}-4 e n\right) / f^{2}$ for some integer $f$.

2.5. The main identities. Here we shall state the main identities, from which the identities in Theorem 1 are derived.

Let the notations $B, \mathcal{O}$, and $X_{6}^{*}$ be defined as in Section 2.1 and $\iota$ be the embedding $B \hookrightarrow M(2, \mathbb{R})$ given by (1). Let the representatives $P_{2}, P_{4}$ and $P_{6}$ of elliptic points of orders 2, 4, 6 be chosen as in (3). Let $t$ be the unique Hauptmodul of $X_{6}^{*}$ that takes values 0,1 , and $\infty$ at $P_{4}, P_{2}$, and $P_{6}$, respectively, and fix a nonzero automorphic form $F(\tau)$ of weight 8 and a nonzero automorphic form $G(\tau)$ of weight 12 on $X_{6}^{*}$.

Let $D$ be the discriminant of an imaginary quadratic order $R_{D}$ with $K=\mathbb{Q}(\sqrt{D})$ such that an optimal embedding $K \hookrightarrow B$ relative to $\left(\mathcal{O}, R_{D}\right)$ exists. Let $\phi: K \hookrightarrow B$ be an optimal embedding relative to $\left(\mathcal{O}, R_{D}\right)$ such that the fixed point $\tau_{0}$ of $\iota\left(\phi\left(K^{*}\right)\right)$ in the upper half-plane lies in the fundamental domain given in Section 2.1 Write $\phi(\sqrt{D})=$ $a_{1} I+a_{2} J+a_{3} I J$ and set

$$
\gamma=\iota(\phi(\sqrt{D}))=\left(\begin{array}{cc}
a_{2} \sqrt{3} & -a_{1}+a_{3} \sqrt{3} \\
a_{1}+a_{3} \sqrt{3} & -a_{2} \sqrt{3}
\end{array}\right) .
$$

Changing $\phi$ to $-\phi$ if necessary, we assume that $a_{1}+a_{3} \sqrt{3}>0$. Set

$$
\widetilde{F}(\tau)=\left.F(\tau)\right|_{8} \gamma, \quad \widetilde{G}(\tau)=\left.G(\tau)\right|_{12} \gamma,
$$

where the slash operator is defined as in (7). 
Now with the settings given as above, our main identities state as follows.

Theorem 2. Set

$$
C_{1}=\frac{4}{\sqrt[4]{12}} \frac{\Gamma(3 / 4)^{2}}{\Gamma(1 / 4)^{2}}, \quad C_{2}=\frac{3}{\sqrt[6]{2}} \frac{\Gamma(2 / 3)^{3}}{\Gamma(1 / 3)^{3}} .
$$

Let $t_{0}=t\left(\tau_{0}\right)$. If $t_{0}$ is real and satisfies $0<t_{0}<1$, then

$$
\sum_{n=0}^{\infty} \frac{(1 / 12)_{n}(1 / 4)_{n}(5 / 12)_{n}}{(1 / 2)_{n}(3 / 4)_{n} n !}\left(8 R t_{0}^{-1} n-R S\right) t_{0}^{n}=\frac{2 a_{3} \sqrt{3}}{\sqrt{|D|}} C_{1}
$$

and

(9) $\quad \sum_{n=0}^{\infty} \frac{(7 / 12)_{n}(3 / 4)_{n}(11 / 12)_{n}}{(3 / 2)_{n}(5 / 4)_{n} n !}\left(8 R t_{0}^{-1}(n+1 / 2)-R S\right) t_{0}^{n}=\frac{2 a_{3} \sqrt{3}}{\sqrt{|D| t_{0}}} C_{1}^{-1}$,

where

(10) $R=\left|t_{0}^{3 / 4}\left(1-t_{0}\right)^{1 / 2}\right|, \quad S=\left.t^{\prime}\left(\tau_{0}\right)^{-1} \frac{d}{d \tau} \log \frac{F(\tau)}{\widetilde{F}(\tau)}\right|_{\tau=\tau_{0}}=\left.\frac{d}{d t} \log \frac{F(\tau)}{\widetilde{F}(\tau)}\right|_{t=t_{0}}$. If $-1<t_{0}<0$, then

$$
\sum_{n=0}^{\infty} \frac{(1 / 12)_{n}(1 / 4)_{n}(5 / 12)_{n}}{(1 / 2)_{n}(3 / 4)_{n} n !}\left(8 R\left|t_{0}\right|^{-1} n+R S\right) t_{0}^{n}=\frac{2 a_{3} \sqrt{6}}{\sqrt{|D|}} C_{1} .
$$

and

$$
\sum_{n=0}^{\infty} \frac{(7 / 12)_{n}(3 / 4)_{n}(11 / 12)_{n}}{(3 / 2)_{n}(5 / 4)_{n} n !}\left(8 R\left|t_{0}\right|^{-1}(n+1 / 2)+R S\right) t_{0}^{n}=\frac{2 a_{3} \sqrt{6}}{\sqrt{\left|D t_{0}\right|}} C_{1}^{-1},
$$

where $R$ and $S$ are defined in the same way as $(10)$.

If $t_{0}$ is real and satisfies $\left|t_{0}\right|>1$, we set $s_{0}=1 / t_{0}$. When $0<s_{0}<1$, we have

$$
\sum_{n=0}^{\infty} \frac{(1 / 12)_{n}(1 / 3)_{n}(7 / 12)_{n}}{(2 / 3)_{n}(5 / 6)_{n} n !}\left(12 R^{\prime} s_{0}^{-1} n-R^{\prime} S^{\prime}\right) s_{0}^{n}=\frac{2 \sqrt{6}\left(a_{2}+a_{3}\right)}{\sqrt{|D|}} C_{2},
$$

and

(14)

$$
\sum_{n=0}^{\infty} \frac{(5 / 12)_{n}(2 / 3)_{n}(11 / 12)_{n}}{(4 / 3)_{n}(7 / 6)_{n} n !}\left(12 R^{\prime} s_{0}^{-1}(n+1 / 2)-R^{\prime} S^{\prime}\right) s_{0}^{n}=\frac{2 \sqrt{6}\left(a_{2}+a_{3}\right)}{\sqrt{|D| s_{0}}} C_{2}^{-1},
$$

where

(15) $\quad R^{\prime}=\left|s_{0}^{5 / 6}\left(1-s_{0}\right)^{1 / 2}\right|, \quad S^{\prime}=\left.s^{\prime}\left(\tau_{0}\right)^{-1} \frac{d}{d \tau} \log \frac{G(\tau)}{\widetilde{G}(\tau)}\right|_{\tau=\tau_{0}}=\left.\frac{d}{d s} \log \frac{G(\tau)}{\widetilde{G}(\tau)}\right|_{s=s_{0}}$.

When $-1<s_{0}<0$, we have

$$
\sum_{n=0}^{\infty} \frac{(1 / 12)_{n}(1 / 3)_{n}(7 / 12)_{n}}{(2 / 3)_{n}(5 / 6)_{n} n !}\left(12 R^{\prime}\left|s_{0}\right|^{-1} n+R^{\prime} S^{\prime}\right) s_{0}^{n}=\frac{2 \sqrt{2}\left(a_{1}-3 a_{3}\right)}{\sqrt{|D|}} C_{2},
$$

and

(17)

$$
\sum_{n=0}^{\infty} \frac{(5 / 12)_{n}(2 / 3)_{n}(11 / 12)_{n}}{(4 / 3)_{n}(7 / 6)_{n} n !}\left(12 R^{\prime}\left|s_{0}\right|^{-1}(n+1 / 2)+R^{\prime} S^{\prime}\right) s_{0}^{n}=\frac{2 \sqrt{2}\left(a_{1}-3 a_{3}\right)}{|D|^{1 / 2}\left|s_{0}\right|^{1 / 3}} C_{2}^{-1},
$$

where $R^{\prime}$ and $S^{\prime}$ are defined as [15].

We will prove the main identities in the next section. 


\section{Proof of THE MAIN IDENTITIES (Theorem 2)}

Let $D<0$ be the discriminant of an imaginary quadratic order $R_{D}$ such that an optimal embedding $\phi$ of relative to $\left(\mathcal{O}, R_{D}\right)$ exists. Let $\alpha=\phi(\sqrt{D})$ and $\gamma=\iota(\alpha)$ so that the fixed point $\tau_{0}$ of $\gamma$ is a CM-point of discriminant $D$ on $X_{6}^{*}$. By conjugating by a suitable element of $\Gamma^{*}$, we may assume that $\tau_{0}$ lies in the fundamental domain described in Section 2.1. Write $\gamma=\left(\begin{array}{ll}a & b \\ c & d\end{array}\right)$. Changing $\phi$ to $-\phi$ if necessary, we assume that $c>0$.

Let $t$ be the Hauptmodul of $X_{6}^{*}$ that takes values 0,1 , and $\infty$ at the elliptic points of orders 4,2 , and 6 , respectively. Let

$$
F_{1}(t)={ }_{2} F_{1}\left(\frac{1}{24}, \frac{5}{24} ; \frac{3}{4} ; t\right), \quad F_{2}(t)=t^{1 / 4}{ }_{2} F_{1}\left(\frac{7}{24}, \frac{11}{24} ; \frac{5}{4} ; t\right),
$$

By Lemma 4 the one-dimensional space $S_{8}\left(X_{6}^{*}\right)$ is spanned by

$$
F(\tau)=\left(F_{1}(t)-C F_{2}(t)\right)^{8}, \quad C=-\frac{4}{\sqrt[4]{12}} \frac{\Gamma(3 / 4)^{2}}{\Gamma(1 / 4)^{2}} .
$$

(Note that $C=-C_{1}$.) Let

$$
\widetilde{F}(\tau)=\left.F(\tau)\right|_{8} \gamma=\frac{(\operatorname{det} \gamma)^{4}}{(c \tau+d)^{8}} F\left(\frac{a \tau+b}{c \tau+d}\right) .
$$

Consider

$$
\frac{d}{d \tau} \log \frac{F(\tau)}{\widetilde{F}(\tau)}=\frac{F^{\prime}(\tau)}{F(\tau)}-\frac{\operatorname{det} \gamma}{(c \tau+d)^{2}} \frac{F^{\prime}(\gamma \tau)}{F(\gamma \tau)}+\frac{8 c}{c \tau+d}
$$

and evaluate the two sides at

$$
\tau=\tau_{0}=\frac{a-d+\sqrt{(a-d)^{2}+4 b c}}{2 c} .
$$

We have

$$
\operatorname{det} \gamma=|D|, \quad c \tau_{0}+d=\frac{a+d+\sqrt{(a+d)^{2}-4 \operatorname{det} \gamma}}{2}=\sqrt{D} .
$$

(Note that $a+d=\operatorname{tr} \gamma=0$.) Thus,

$$
\left.\frac{d}{d \tau} \log \frac{F(\tau)}{\widetilde{F}(\tau)}\right|_{\tau=\tau_{0}}=2 \frac{F^{\prime}\left(\tau_{0}\right)}{F\left(\tau_{0}\right)}+\frac{8 c}{\sqrt{D}} .
$$

Set $t_{0}=t\left(\tau_{0}\right)$ and assume that $\left|t_{0}\right|<1$. For the right-hand side of (19), we have, by (18),

$$
\frac{F^{\prime}\left(\tau_{0}\right)}{F\left(\tau_{0}\right)}=\left.\frac{8}{F_{1}\left(t_{0}\right)-C F_{2}\left(t_{0}\right)} \frac{d}{d \tau}\left(F_{1}-C F_{2}\right)\right|_{\tau=\tau_{0}} .
$$

Set

$$
u=\frac{\tau_{0}-P_{4}}{\tau_{0}-\bar{P}_{4}}=\frac{\tau_{0}-i}{\tau_{0}+i} .
$$

Then, by Lemma 3 , we have

$$
C F_{2}\left(t_{0}\right)=u F_{1}\left(t_{0}\right) \text {. }
$$

Also, differentiating the two sides of (4) in Lemma 3 with respect to $\tau$ and then evaluating at $\tau_{0}$, we obtain

$$
\begin{aligned}
\frac{2 i}{\left(\tau_{0}+i\right)^{2}} & =\left.\frac{1}{F_{1}\left(t_{0}\right)^{2}}\left(C F_{1} \frac{d}{d \tau} F_{2}-C F_{2} \frac{d}{d \tau} F_{1}\right)\right|_{\tau=\tau_{0}} \\
& =\left.\frac{1}{F_{1}\left(t_{0}\right)}\left(C \frac{d}{d \tau} F_{2}-u \frac{d}{d \tau} F_{1}\right)\right|_{\tau=\tau_{0}} .
\end{aligned}
$$


That is,

$$
\left.C \frac{d}{d \tau} F_{2}\right|_{\tau=\tau_{0}}=\left.u \frac{d}{d \tau} F_{1}\right|_{\tau=\tau_{0}}+\frac{2 i}{\left(\tau_{0}+i\right)^{2}} F_{1}\left(t_{0}\right) .
$$

Substituting this and 22, into 20, we get

$$
\begin{aligned}
\frac{F^{\prime}\left(\tau_{0}\right)}{F\left(\tau_{0}\right)} & =\frac{8}{(1-u) F_{1}\left(t_{0}\right)}\left(\left.(1-u) \frac{d F_{1}}{d \tau}\right|_{\tau=\tau_{0}}-\frac{2 i}{\left(\tau_{0}+i\right)^{2}} F_{1}\left(t_{0}\right)\right) \\
& =\left.\frac{8}{F_{1}\left(t_{0}\right)} \frac{d F_{1}}{d \tau}\right|_{\tau=\tau_{0}}-\frac{8}{\tau_{0}+i} .
\end{aligned}
$$

By (5) in Lemma 3, we find

$$
\begin{aligned}
\left.\frac{d F_{1}}{d \tau}\right|_{\tau=\tau_{0}} & =\left.\frac{d F_{1}(t)}{d t}\right|_{t=t_{0}} t^{\prime}\left(\tau_{0}\right)=\left.\frac{2 t_{0}^{3 / 4}\left(1-t_{0}\right)^{1 / 2}}{C i}\left(F_{1}\left(t_{0}\right)-C F_{2}\left(t_{0}\right)\right)^{2} \frac{d F_{1}(t)}{d t}\right|_{t=t_{0}} \\
& =\left.\frac{2 R(1-u)^{2}}{C i} F_{1}\left(t_{0}\right)^{2} \frac{d F_{1}(t)}{d t}\right|_{t=t_{0}},
\end{aligned}
$$

where

$$
R=t_{0}^{3 / 4}\left(1-t_{0}\right)^{1 / 2}
$$

Plugging this into 24, we arrive at

$$
\frac{F^{\prime}\left(\tau_{0}\right)}{F\left(\tau_{0}\right)}=\left.\frac{16 R(1-u)^{2}}{C i} F_{1}\left(t_{0}\right) \frac{d F_{1}(t)}{d t}\right|_{t=t_{0}}-\frac{8}{\tau_{0}+i} .
$$

For the left-hand side of (19), we have, by (3) in Lemma 3 again,

$$
\left.\frac{d}{d \tau} \log \frac{F(\tau)}{\widetilde{F}(\tau)}\right|_{\tau=\tau_{0}}=\left.\frac{d}{d t} \log \frac{F(\tau)}{\widetilde{F}(\tau)}\right|_{t=t_{0}} \cdot \frac{2 R}{C i}\left(F_{1}\left(t_{0}\right)-C F_{2}\left(t_{0}\right)\right)^{2}
$$

Observe that $F(\tau) / \widetilde{F}(\tau)$ is an automorphic function on $\Gamma^{*} \cap\left(\gamma^{-1} \Gamma^{*} \gamma\right)$, which is a subgroup of finite index in $\Gamma^{*}$. Therefore, $F(\tau) / \widetilde{F}(\tau)$ is an algebraic function of $t$, and so is $d \log (F(\tau) / \widetilde{F}(\tau)) / d t$. Let

$$
S=\left.\frac{d}{d t} \log \frac{F(\tau)}{\widetilde{F}(\tau)}\right|_{t=t_{0}}
$$

Then 27) becomes

$$
\left.\frac{d}{d \tau} \log \frac{F(\tau)}{\widetilde{F}(\tau)}\right|_{\tau=\tau_{0}}=\frac{2 R S}{C i}(1-u)^{2} F_{1}\left(t_{0}\right)^{2} .
$$

Combining this with (19) and (26), we obtain

$$
\frac{2 R S}{C i}(1-u)^{2} F_{1}\left(t_{0}\right)^{2}=\left.\frac{32 R}{C i}(1-u)^{2} F_{1}\left(t_{0}\right) \frac{d F_{1}(t)}{d t}\right|_{t=t_{0}}-\frac{16}{\tau_{0}+i}+\frac{8 c}{\sqrt{D}} .
$$

Write $\phi(\sqrt{D})=a_{1} I+a_{2} J+a_{3} I J$ so that

$$
\gamma=\left(\begin{array}{cc}
a_{2} \sqrt{3} & -a_{1}+a_{3} \sqrt{3} \\
a_{1}+a_{3} \sqrt{3} & -a_{2} \sqrt{3}
\end{array}\right), \quad \tau_{0}=\frac{a_{2} \sqrt{3}+\sqrt{D}}{a_{1}+a_{3} \sqrt{3}} .
$$


Then

$$
\begin{aligned}
(1-u)^{-2} & \left(\frac{16}{\tau_{0}+i}-\frac{8 c}{\sqrt{D}}\right)=-\frac{\left(\tau_{0}+i\right)^{2}}{4}\left(\frac{16}{\tau_{0}+i}-\frac{8\left(a_{1}+a_{3} \sqrt{3}\right)}{\sqrt{D}}\right) \\
& =-2\left(\tau_{0}+i\right) \frac{a_{1}+a_{3} \sqrt{3}}{\sqrt{D}}\left(\frac{2 \sqrt{D}}{a_{1}+a_{3} \sqrt{3}}-\frac{a_{2} \sqrt{3}+\sqrt{D}}{a_{1}+a_{3} \sqrt{3}}-i\right) \\
& =\frac{2\left(a_{1}+a_{3} \sqrt{3}\right)}{\sqrt{D}}\left(\tau_{0}+i\right)\left(\bar{\tau}_{0}+i\right) .
\end{aligned}
$$

Now it is necessary to consider the two cases $0<t_{0}<1$ and $-1<t_{0}<0$ separately.

Assume first $0<t_{0}<1$. By Lemma 5, we have $a_{2}=0$ and

$$
\gamma=\left(\begin{array}{cc}
0 & -a_{1}+a_{3} \sqrt{3} \\
a_{1}+a_{3} \sqrt{3} & 0
\end{array}\right), \quad \tau_{0}=\frac{\sqrt{D}}{a_{1}+a_{3} \sqrt{3}} .
$$

Thus, from 30 , we get

$$
(1-u)^{-2}\left(\frac{16}{\tau_{0}+i}-\frac{8 c}{\sqrt{D}}\right)=\frac{2\left(a_{1}+a_{3} \sqrt{3}\right)}{\sqrt{D}}\left(\frac{|D|}{\left(a_{1}+a_{3} \sqrt{3}\right)^{2}}-1\right)
$$

Now det $\gamma=|D|$ implies that $|D|=\left(a_{1}+a_{3} \sqrt{3}\right)\left(a_{1}-a_{3} \sqrt{3}\right)$. It follows that

$$
(1-u)^{-2}\left(\frac{16}{\tau_{0}+i}-\frac{8 c}{\sqrt{D}}\right)=\frac{2\left(a_{1}+a_{3} \sqrt{3}\right)}{\sqrt{D}}\left(\frac{a_{1}-a_{3} \sqrt{3}}{a_{1}+a_{3} \sqrt{3}}-1\right)=-\frac{4 a_{3} \sqrt{3}}{i \sqrt{|D|}} .
$$

Then we deduce from this and 29 that

$$
\left.16 R F_{1}\left(t_{0}\right) \frac{d F_{1}(t)}{d t}\right|_{t=t_{0}}-R S F_{1}\left(t_{0}\right)^{2}=-\frac{2 a_{3} \sqrt{3}}{\sqrt{|D|}} C,
$$

where $R$ and $S$ are the numbers defined in 25 and (28), respectively.

Furthermore, from (5) and (23), we have

$$
\begin{aligned}
\left.C \frac{d F_{2}(t)}{d t}\right|_{t=t_{0}} & =\left.u \frac{d F_{1}(t)}{d t}\right|_{t=t_{0}}+\frac{2 i F_{1}\left(t_{0}\right)}{\left(\tau_{0}+i\right)^{2}} \frac{C i}{2 R\left(F_{1}\left(t_{0}\right)-C F_{2}\left(t_{0}\right)\right)^{2}} \\
& =\left.u \frac{d F_{1}(t)}{d t}\right|_{t=t_{0}}+\frac{2 i}{\left(\tau_{0}+i\right)^{2}} \frac{C i}{2 R(1-u)^{2} F_{1}\left(t_{0}\right)} \\
& =\left.u \frac{d F_{1}(t)}{d t}\right|_{t=t_{0}}+\frac{C}{4 R F_{1}\left(t_{0}\right)} .
\end{aligned}
$$

From this, 222), and (32), it follows that

$$
16 R \frac{C}{u} F_{2}\left(t_{0}\right)\left(\left.\frac{C}{u} \frac{d F_{2}(t)}{d t}\right|_{t=t_{0}}-\frac{C}{4 R u F_{1}\left(t_{0}\right)}\right)-R S \frac{C^{2}}{u^{2}} F_{2}\left(t_{0}\right)^{2}=-\frac{2 a_{3} \sqrt{3}}{\sqrt{|D|}} C .
$$

Equivalently, we have

$$
\left.16 R F_{2}\left(t_{0}\right) \frac{d F_{2}(t)}{d t}\right|_{t=t_{0}}-R S F_{2}\left(t_{0}\right)^{2}=\left(4 u-\frac{2 u^{2} a_{3} \sqrt{3}}{\sqrt{|D|}}\right) C^{-1}
$$


Now from (21), 31], and the fact $|D|=a_{1}^{2}-3 a_{3}^{2}$, we have

$$
\begin{aligned}
u & =\frac{\sqrt{|D|}-a_{1}-a_{3} \sqrt{3}}{\sqrt{|D|}+a_{1}+a_{3} \sqrt{3}}=\frac{\left(\sqrt{|D|}-a_{1}-a_{3} \sqrt{3}\right)^{2}}{|D|-\left(a_{1}+a_{3} \sqrt{3}\right)^{2}} \\
& =\frac{a_{1}^{2}-3 a_{3}^{2}-2\left(a_{1}+a_{3} \sqrt{3}\right) \sqrt{|D|}+\left(a_{1}+a_{3} \sqrt{3}\right)^{2}}{-2 a_{1} a_{3} \sqrt{3}-6 a_{3}^{2}}=-\frac{a_{1}-\sqrt{|D|}}{a_{3} \sqrt{3}}
\end{aligned}
$$

so that

$$
\begin{aligned}
4 u-\frac{2 u^{2} a_{3} \sqrt{3}}{\sqrt{|D|}} & =\frac{a_{1}-\sqrt{|D|}}{a_{3} \sqrt{3}}\left(-4-2 \frac{a_{1}-\sqrt{|D|}}{\sqrt{|D|}}\right) \\
& =-2 \frac{\left(a_{1}-\sqrt{|D|}\right)\left(a_{1}+\sqrt{|D|}\right)}{a_{3} \sqrt{3|D|}}=-\frac{2 a_{3} \sqrt{3}}{\sqrt{|D|}} .
\end{aligned}
$$

Substituting this into (34), we get

$$
\left.16 R F_{2}\left(t_{0}\right) \frac{d F_{2}(t)}{d t}\right|_{t=t_{0}}-R S F_{2}\left(t_{0}\right)^{2}=-\frac{2 a_{3} \sqrt{3}}{C \sqrt{|D|}} .
$$

Now we consider the cases $-1<t_{0}<0$. By Lemma 5, we have $a_{2}=-a_{3}$ so that

$$
\gamma=\left(\begin{array}{cc}
-a_{3} \sqrt{3} & -a_{1}+a_{3} \sqrt{3} \\
a_{1}+a_{3} \sqrt{3} & a_{3} \sqrt{3}
\end{array}\right), \quad \tau_{0}=\frac{-a_{3} \sqrt{3}+\sqrt{D}}{a_{1}+a_{3} \sqrt{3}} .
$$

In this case, we have $|D|=a_{1}^{2}-6 a_{3}^{2}$ and 30 becomes

$$
\begin{aligned}
(1-u)^{-2}\left(\frac{16}{\tau_{0}+i}-\frac{8 c}{\sqrt{D}}\right) & =\frac{2\left(a_{1}+a_{3} \sqrt{3}\right)}{\sqrt{D}}\left(\frac{a_{1}^{2}-3 a_{3}^{2}}{\left(a_{1}+a_{3} \sqrt{3}\right)^{2}}-\frac{2 a_{3} \sqrt{3} i}{a_{1}+a_{3} \sqrt{3}}-1\right) \\
& =-\frac{4 a_{3} \sqrt{3}}{\sqrt{D}}(1+i)=-\frac{4 a_{3} \sqrt{6}}{i \sqrt{|D|}} e^{2 \pi i / 8} .
\end{aligned}
$$

Instead of 32, we now have

$$
\left.16 R F_{1}\left(t_{0}\right) \frac{d F_{1}(t)}{d t}\right|_{t=t_{0}}-R S F_{1}\left(t_{0}\right)^{2}=-\frac{2 a_{3} \sqrt{6}}{\sqrt{|D|}} e^{2 \pi i / 8} C .
$$

Likewise, instead of 33, we have

$$
16 R \frac{C}{u} F_{2}\left(t_{0}\right)\left(\left.\frac{C}{u} \frac{d F_{2}(t)}{d t}\right|_{t=t_{0}}-\frac{C}{4 R u F_{1}\left(t_{0}\right)}\right)-R S \frac{C^{2}}{u^{2}} F_{2}\left(t_{0}\right)^{2}=-\frac{2 a_{3} \sqrt{6}}{\sqrt{|D|}} e^{2 \pi i / 8} C,
$$

or equivalently,

$$
\left.16 R F_{2}\left(t_{0}\right) \frac{d F_{2}(t)}{d t}\right|_{t=t_{0}}-R S F_{2}\left(t_{0}\right)^{2}=\left(4 u-\frac{2 u^{2} a_{3} \sqrt{6}}{\sqrt{|D|}} e^{2 \pi i i / 8}\right) C^{-1} .
$$

Now

$$
\begin{aligned}
u & =\frac{-a_{3} \sqrt{3}+i\left(\sqrt{|D|}-a_{1}-a_{3} \sqrt{3}\right)}{-a_{3} \sqrt{3}+i\left(\sqrt{|D|}+a_{1}+a_{3} \sqrt{3}\right)}=\frac{\left(a_{3} \sqrt{3}+i\left(a_{1}+a_{3} \sqrt{3}\right)\right)^{2}+|D|}{3 a_{3}^{2}+\left(\sqrt{|D|}+a_{1}+a_{3} \sqrt{3}\right)^{2}} \\
& =\frac{-2 a_{1} a_{3} \sqrt{3}-6 a_{3}^{2}+2 a_{3} \sqrt{3}\left(a_{1}+a_{3} \sqrt{3}\right) i}{2 a_{1}^{2}+2 a_{1} a_{3} \sqrt{3}+2\left(a_{1}+a_{3} \sqrt{3}\right) \sqrt{|D|}} \\
& =\frac{a_{3} \sqrt{3}(-1+i)}{a_{1}+\sqrt{|D|}}=-\frac{a_{1}-\sqrt{|D|}}{a_{3} \sqrt{6}} e^{-2 \pi i / 8}
\end{aligned}
$$


and hence

$$
\begin{aligned}
4 u-\frac{2 u^{2} a_{3} \sqrt{6}}{\sqrt{|D|}} e^{2 \pi i / 8} & =2 u\left(2+\frac{a_{1}-\sqrt{|D|}}{\sqrt{|D|}}\right) \\
& =-2 e^{-2 \pi i / 8} \frac{a_{1}-\sqrt{|D|}}{a_{3} \sqrt{6}} \frac{a_{1}+\sqrt{|D|}}{\sqrt{|D|}}=-2 e^{-2 \pi i / 8} \frac{a_{3} \sqrt{6}}{\sqrt{|D|}}
\end{aligned}
$$

Then (37) becomes

$$
\left.16 R F_{2}\left(t_{0}\right) \frac{d F_{2}(t)}{d t}\right|_{t=t_{0}}-R S F_{2}\left(t_{0}\right)^{2}=-\frac{2 a_{3} \sqrt{6}}{\sqrt{|D|}} e^{-2 \pi i / 8} C^{-1}
$$

Now by Clausen's identity [2]

$$
{ }_{2} F_{1}(\alpha, \beta ; \alpha+\beta+1 / 2 ; z)^{2}={ }_{3} F_{2}(2 \alpha, \alpha+\beta, 2 \beta ; 2 \alpha+2 \beta, \alpha+\beta+1 / 2 ; z),
$$

we may write

$$
\begin{aligned}
F_{1}\left(t_{0}\right)^{2} & ={ }_{2} F_{1}\left(\frac{1}{24}, \frac{5}{24} ; \frac{3}{4} ; t_{0}\right)^{2}={ }_{3} F_{2}\left(\frac{1}{12}, \frac{1}{4}, \frac{5}{12} ; \frac{1}{2}, \frac{3}{4} ; t_{0}\right) \\
& =\sum_{n=0}^{\infty} \frac{(1 / 12)_{n}(1 / 4)_{n}(5 / 12)_{n}}{(1 / 2)_{n}(3 / 4)_{n} n !} t_{0}^{n},
\end{aligned}
$$

and

$$
\left.2 F_{1}\left(t_{0}\right) \frac{d F_{1}(t)}{d t}\right|_{t=t_{0}}=\sum_{n=0}^{\infty} \frac{(1 / 12)_{n}(1 / 4)_{n}(5 / 12)_{n}}{(1 / 2)_{n}(3 / 4)_{n} n !} n t_{0}^{n-1} .
$$

Substituting these two expressions into (32), we find that for the cases $0<t_{0}<1$,

$$
\sum_{n=0}^{\infty} \frac{(1 / 12)_{n}(1 / 4)_{n}(5 / 12)_{n}}{(1 / 2)_{n}(3 / 4)_{n} n !}\left(8 R t_{0}^{-1} n-R S\right) t_{0}^{n}=-\frac{2 a_{3} \sqrt{3}}{\sqrt{|D|}} C .
$$

This proves (8). For the cases $-1<t_{0}<0$, we note that, according to the description of $t^{1 / 4}$ given in Lemma 3 , we have $R=-e^{2 \pi i / 8}|R|$. In these cases, 36 becomes

$$
\sum_{n=0}^{\infty} \frac{(1 / 12)_{n}(1 / 4)_{n}(5 / 12)_{n}}{(1 / 2)_{n}(3 / 4)_{n} n !}\left(8|R|\left|t_{0}\right|^{-1} n+|R| S\right) t_{0}^{n}=-\frac{2 a_{3} \sqrt{6}}{\sqrt{|D|}} C .
$$

This is (11) in the theorem.

Likewise, by (39) again, we have

$$
\begin{aligned}
F_{2}\left(t_{0}\right)^{2} & =t_{0}^{1 / 2}{ }_{2} F_{1}\left(\frac{7}{24}, \frac{11}{24} ; \frac{5}{4} ; t_{0}\right)^{2}=t_{0}^{1 / 2}{ }_{3} F_{2}\left(\frac{7}{12}, \frac{3}{4}, \frac{11}{12} ; \frac{3}{2} ; \frac{5}{4} ; t_{0}\right) \\
& =t_{0}^{1 / 2} \sum_{n=0}^{\infty} \frac{(7 / 12)_{n}(3 / 4)_{n}(11 / 12)_{n}}{(3 / 2)_{n}(5 / 4)_{n} n !} t_{0}^{n}
\end{aligned}
$$

and

$$
\left.2 F_{2}\left(t_{0}\right) \frac{d F_{2}(t)}{d t}\right|_{t=t_{0}}=t_{0}^{1 / 2} \sum_{n=0}^{\infty} \frac{(7 / 12)_{n}(3 / 4)_{n}(11 / 12)_{n}}{(3 / 2)_{n}(5 / 4)_{n} n !}(n+1 / 2) t_{0}^{n-1}
$$

Substituting these two into (35), we obtain, for the cases $0<t_{0}<1$,

$$
\sum_{n=0}^{\infty} \frac{(7 / 12)_{n}(3 / 4)_{n}(11 / 12)_{n}}{(3 / 2)_{n}(5 / 4)_{n} n !}\left(8 R t_{0}^{-1}(n+1 / 2)-R S\right) t_{0}^{n}=-\frac{2 a_{3} \sqrt{3}}{\sqrt{|D| t_{0}}} C^{-1} \text {. }
$$


This proves 99. For the cases $-1<t_{0}<0$, we have $t_{0}^{1 / 2}=e^{-2 \pi i / 4}\left|t_{0}\right|$ and $R=$ $-e^{2 \pi i / 8}|R|$, and (38) yields

$$
\sum_{n=0}^{\infty} \frac{(7 / 12)_{n}(3 / 4)_{n}(11 / 12)_{n}}{(3 / 2)_{n}(5 / 4)_{n} n !}\left(8|R|\left|t_{0}\right|^{-1}(n+1 / 2)+|R| S\right) t_{0}^{n}=-\frac{2 a_{3} \sqrt{6}}{\sqrt{\left|D t_{0}\right|}} C^{-1} .
$$

This is $(12)$ in the theorem.

The proof of the identities $(13)-(17)$ is very similar to the proof of $(8)-(12)$ and is skipped.

\section{Proof of Theorem 1}

Let $t(\tau)$ be the Hauptmodul of $X_{6}^{*}$ that takes values 0,1 , and $\infty$ at the elliptic points of order 4,2 , and 6 , respectively. There are finitely many discriminants $D$ such that the number of CM-point of discriminant $D$ on $X_{6}^{*}$ is one. These discriminants are given in the first columns of the tables in Theorem 1 and Remark 1 . The values of $t$ at these CMpoints were determined numerically by Elkies [12] and later proved rigorously by Errthum [13] using Borcherds forms. These values are given by $M / N$, where $M$ and $N$ are the integers from the tables. Now according to Theorem 2, to prove the identity associated to the discriminant $D$, the main task is the evaluation of the constant $S$ in (10) or $S^{\prime}$ in (15). Here we will work out two cases and omit the others.

Let $D=-120$ and choose the optimal embedding $\phi: \mathbb{Q}(\sqrt{-30}) \rightarrow B$ relative to $(\mathcal{O}, \mathbb{Z}[\sqrt{-30}])$ to be the one determined by $\phi(\sqrt{-30})=6 I-J+I J$. Let

$$
\gamma=\iota(\phi(\sqrt{-120}))=2\left(\begin{array}{cc}
-\sqrt{3} & -6+\sqrt{3} \\
6+\sqrt{3} & \sqrt{3}
\end{array}\right)
$$

and $\tau_{0}=(-\sqrt{3}+\sqrt{-30}) /(6+\sqrt{3})$ be the fixed point of $\gamma$ in the upper half-plane. Then $\tau_{0}$ is the representative of the CM-point of discriminant -120 in the fundamental domain described in Section 2.1. According to Theorem 2, we need to evaluate the function

$$
f(\tau)=t^{\prime}(\tau)^{-1} \frac{d}{d \tau} \log \frac{F(\tau)}{\widetilde{F}(\tau)}=\frac{d}{d t} \log \frac{F(\tau)}{\widetilde{F}(\tau)}
$$

at $\tau_{0}$, where $F(\tau)$ is any nonzero automorphic form of weight 8 on $X_{6}^{*}$ and $\widetilde{F}(\tau)=$ $\left.F(\tau)\right|_{8} \gamma$. In order to do so, we note that $g(\tau)$ is an automorphic function on the subgroup $\Gamma^{*} \cap\left(\gamma^{-1} \Gamma^{*} \gamma\right)$ and hence $t(\tau)$ and $g(\tau)$ satisfy a polynomial relation $P(t, f)=0$ for some polynomial $P(x, z)$. Then $f\left(\tau_{0}\right)$ will be a root of the polynomial $P\left(t\left(\tau_{0}\right), z\right)$ in $z$.

Here, to determine the polynomial $P(x, y)$, we observe that the matrix $\gamma_{0}=\gamma / \sqrt{6}$ lies in $\Gamma_{5}^{*}$, where $\Gamma_{p}^{*}$ is defined by (6), and can be taken to be one of the coset representatives in $\Gamma^{*} \backslash \Gamma_{5}^{*}$ defining the 5 th Hecke operator $T_{5}$ on $X_{6}^{*}$. Let $\gamma_{1}, \ldots, \gamma_{5}$ be the other coset representative defining $T_{5}$. Then any symmetric sum of $\left.F(\tau)\right|_{8} \gamma_{j}$ will be an automorphic function on $X_{6}^{*}$ and hence equal to a rational function in $t$. In particular, there is a polynomial $Q(x, y)$ of degree 6 in $y$ such that $Q(t, F / \widetilde{F})=0$. Then we have

$$
\begin{aligned}
0 & =\frac{d}{d \tau} Q(t(\tau), F(\tau) / \widetilde{F}(\tau)) \\
& =\left.t^{\prime}(\tau) \frac{\partial}{\partial x} Q(x, y)\right|_{x=t(\tau), y=F(\tau) / \widetilde{F}(\tau)}+\left.\left(\frac{F(\tau)}{\widetilde{F}(\tau)}\right)^{\prime} \frac{\partial}{\partial y} Q(x, y)\right|_{x=t(\tau), y=F(\tau) / \widetilde{F}(\tau)} .
\end{aligned}
$$


Eliminating the variable $y$ from the two polynomials

$$
Q(x, y), \quad \frac{\partial}{\partial x} Q(x, y)+y z \frac{\partial}{\partial y} Q(x, y),
$$

we get the polynomial $P(x, z)$ satisfying $P(t, f)=0$.

From the above discussion, we see that the problem of proving the identity for the discriminant -120 boils down to the determination of the polynomial $Q(x, y)$ satisfying $Q(t, F / \widetilde{F})=0$. This is where the method of computing Hecke operators developed in [18] comes in.

Let $u=-540 / t$ be the Hauptmodul of $X_{6}^{*}$ that takes values $0,-540$, and $\infty$ at $P_{6}, P_{2}$, and $P_{4}$, respectively. By Equation (16) of [18], for a positive even integer $k$, a basis for the space of automorphic forms of weight $k$ on $X_{6}^{*}$ is given by

$$
\begin{aligned}
g_{k, \ell}= & u^{\{5 k / 12\}}(1+u / 540)^{\{k / 4\}} u^{\ell} \\
& \times\left({ }_{2} F_{1}\left(\frac{1}{24}, \frac{7}{24} ; \frac{5}{6} ;-\frac{u}{540}\right)-C^{\prime} u^{1 / 6}{ }_{2} F_{1}\left(\frac{5}{24}, \frac{11}{24} ; \frac{7}{6} ;-\frac{u}{540}\right)\right)^{k},
\end{aligned}
$$

$\ell=0, \ldots, d_{k}-1=\lfloor 5 k / 12\rfloor+\lfloor l / 4\rfloor+\lfloor 3 k / 8\rfloor-k$. In Section 4 of [18], we discussed how to compute Hecke operators with respect to these bases. The results relevant to our problem here are given in the following table.

\begin{tabular}{r|ll}
\hline \hline$k$ & $M$ & \\
\hline 8 & -114 & \\
16 & 77646 & \\
24 & $\left(\begin{array}{cc}10980750 & 3111696 / 5 \\
55987200000 & 14267406\end{array}\right)$ \\
32 & $\left(\begin{array}{cc}105068988750 & 376515216 / 5 \\
12317184000000 & -39127734834\end{array}\right)$ \\
40 & $\left(\begin{array}{cc}-70619784011250 & 45558341136 / 5 \\
30930128640000000 & 36422537206926\end{array}\right)$ & \\
48 & $\left(\begin{array}{ccc}100480725468750 & 225950546273760 & 2420662999104 / 5 \\
512317872000000000 & 22159766272716750 & 5512559277456 / 5 \\
2612138803200000000000 & -7950573190656000000 & -23013714467131314\end{array}\right)$ \\
\hline \hline
\end{tabular}

That is, we have

$$
T_{5}\left(\begin{array}{c}
g_{k, 0} \\
\vdots \\
g_{k, d_{k}-1}
\end{array}\right)=M\left(\begin{array}{c}
g_{k, 0} \\
\vdots \\
g_{k, d_{k}-1}
\end{array}\right) .
$$

Now we choose the nonzero automorphic form $F$ of weight 8 to be $g_{8,0}$. From the description of $g_{k, \ell}$, we find

$$
F^{k / 8}=g_{k, d_{k}-1}
$$

for any weight $k$ that is a multiple of 8 . Then the table above gives us

$$
T_{5} F=-114 F, \quad T_{5} F^{2}=77646 F^{2}
$$




$$
\begin{aligned}
& T_{5} F^{3}=(55987200000 / u+14267406) F^{3}=(-103680000 t+14267406) F^{3}, \\
& T_{5} F^{4}=(-22809600000 t-39127734834) F^{4}, \\
& T_{5} F^{5}=(-57278016000000 t+36422537206926) F^{5}, \\
& T_{5} F^{6}=\left(8957952000000000 t^{2}+14723283686400000 t-23013714467131314\right) F^{6} .
\end{aligned}
$$

By the definition of Hecke operators, we have

$$
T_{5} F^{\ell}=\left.5^{4 \ell-1} \sum_{j=0}^{5} F^{\ell}\right|_{8 \ell} \gamma_{j} .
$$

Then an application of Newton's identity yields

$$
\begin{aligned}
\prod_{j=0}^{5}\left(1-\frac{\left.F\right|_{8} \gamma_{j}}{F} y\right)=1 & +\frac{114}{125} y-\frac{6333}{78125} y^{2}+\frac{4}{5^{11}}(8640000 t-5177953) y^{3} \\
& +\frac{3}{5^{15}}(8467200000 t+1804020097) y^{4} \\
& +\frac{726}{5^{20}}(93744000000 t-3501556201) y^{5} \\
& +\frac{1}{5^{16}}(138240 t+14641)^{2} y^{6} .
\end{aligned}
$$

Replacing $t$ by $x$ in the last expression, we get the polynomial $Q(x, y)$. Computing the resultant of the two polynomials in 40 with respect to $y$, we find that the polynomial $P(x, z)$ is

$$
\begin{array}{rl}
P(x, z)=2 & 7 x^{3}(x-1)^{2}(138240 x+14641) z^{6}+7464960 x^{3}(x-1)^{2} z^{5} \\
& +1080 x^{2}(x-1)(5760 x-5041) z^{4}+8640 x(x-1)(320 x-183) z^{3} \\
& +2160\left(320 x^{2}-363 x+75\right) z^{2}+18432(5 x-3) z+5120 .
\end{array}
$$

By [12, 13], we know that $t\left(\tau_{0}\right)=-7^{4} / 15^{3}$ and consequently, $f\left(\tau_{0}\right)$ is equal to one of the six zeros of the polynomial $P\left(-7^{4} / 15^{3}, z\right)$ in $z$. To determine which zero is $f\left(\tau_{0}\right)$, we note that the polynomial $P(x, z)$ can actually be taken as a defining equation of the Shimura curve $X_{6}(5) / W_{6}$ over $\mathbb{Q}$, where $X_{6}(5)$ denotes the Shimura curve associated to the Eichler order $\mathcal{O} \cap\left(\gamma^{-1} \mathcal{O} \gamma\right)$ of level 5 and $X_{6}(5) / W_{6}$ denotes its quotient by the Atkin-Lehner involution $w_{2}$ and $w_{3}$. Since $\tau_{0}$ as a point on $X_{6}^{*}(5)$ is the unique CM-point of discriminant -120 , it is a rational point on $X_{6}^{*}(5)$ over $\mathbb{Q}$. In other words, the only rational zero $2250 / 6517$ of the polynomial $P\left(-7^{4} / 15^{3}, z\right)$ must be the value of $f\left(\tau_{0}\right)$. (The other five zeros correspond to the CM-points of discriminant $-5^{2} \cdot 120$ on $X_{6}^{*}(5)$.) Thus, the two numbers $R$ and $S$ in $(10)$ in the case of $D=-120$ are

and (11) and (12) become

$$
R=\frac{2^{2} \cdot 7^{3} \cdot 19}{15^{15 / 4}}, \quad S=\frac{2250}{6517}
$$

$$
\frac{2^{2} \cdot 7^{3} \cdot 19}{15^{5 / 4}} \sum_{n=0}^{\infty}\left(8 \cdot \frac{15^{3}}{7^{4}} n+\frac{2250}{6517}\right) A_{n}\left(-\frac{7^{4}}{15^{3}}\right)^{n}=\frac{2}{\sqrt{5}} C_{1}
$$

and

$$
\frac{2^{2} \cdot 7^{3} \cdot 19}{15^{5 / 4}} \sum_{n=0}^{\infty}\left(8 \cdot \frac{15^{3}}{7^{4}}(n+1 / 2)+\frac{2250}{6517}\right) A_{n}^{\prime}\left(-\frac{7^{4}}{15^{3}}\right)^{n}=\frac{30 \sqrt{3}}{49} C_{1}^{-1} .
$$

This proves the identities for the discriminant -120 . 
We next consider the case $D=-19$. Choose the optimal embedding $\phi: \mathbb{Q}(\sqrt{-19}) \hookrightarrow$ $B$ relative to $(\mathcal{O}, \mathbb{Z}[(1+\sqrt{-19}) / 2])$ to be the one determined by $\phi(\sqrt{-19})=5 I-J+$ $I J$. Let $\gamma_{0}=\iota(\phi(\sqrt{-19}))$ and write it as $\gamma_{0}=\left(\begin{array}{ll}a & b \\ c & d\end{array}\right)$. Its fixed point $\tau_{0}=(-\sqrt{3}+$ $\sqrt{-19}) /(5+\sqrt{3})$ in the upper half-plane lies in the fundamental domain. The main task is to find the value of

$$
S^{\prime}=s^{\prime}(\tau)^{-1} \frac{d}{d \tau} \log \frac{G(\tau)}{\widetilde{G}(\tau)}
$$

in (15) at $\tau_{0}$, where $G(\tau)$ is any nonzero automorphic form of weight 12 on $X_{6}^{*}, \widetilde{G}=$ $\left.G\right|_{12} \gamma_{0}$, and $s(\tau)=1 / t(\tau)$. By Lemma 4 , we know that we can choose $G=t^{1 / 2} F^{3 / 2}$. Thus,

$$
\begin{aligned}
S^{\prime} & =-t^{\prime}\left(\tau_{0}\right)^{-1} t\left(\tau_{0}\right)^{2}\left(\left.\frac{3}{2} \frac{d}{d \tau} \log \frac{F(\tau)}{\widetilde{F}(\tau)}\right|_{\tau=\tau_{0}}+\frac{t^{\prime}\left(\tau_{0}\right)}{2 t\left(\tau_{0}\right)}-\frac{19}{\left(c \tau_{0}+d\right)^{2}} \frac{t^{\prime}\left(\gamma_{0} \tau_{0}\right)}{t\left(\gamma_{0} \tau_{0}\right)}\right) \\
& =-\left.\frac{3}{2} t^{\prime}\left(\tau_{0}\right)^{-1} t\left(\tau_{0}\right)^{2} \frac{d}{d \tau} \log \frac{F(\tau)}{\widetilde{F}(\tau)}\right|_{\tau=\tau_{0}}-t\left(\tau_{0}\right),
\end{aligned}
$$

where $\widetilde{F}=\left.F\right|_{8} \gamma_{0}$.

Now observe that

$$
\gamma_{1}:=\iota\left(\phi\left(\frac{1+\sqrt{-19}}{2}\right)\right)=\frac{1}{2}\left(I_{2}+\gamma_{0}\right)=\frac{1}{2}\left(\begin{array}{cc}
1+a & b \\
c & 1+d
\end{array}\right) .
$$

has determinant 5 and can be taken to be a coset representative for $\Gamma^{*} \backslash \Gamma_{5}^{*}$ defining the Hecke operator $T_{5}$. Therefore, letting $\widetilde{F}_{1}(\tau)=\left.F(\tau)\right|_{8} \gamma_{1}$ and

$$
f_{1}(\tau)=t^{\prime}(\tau) \frac{d}{d \tau} \log \frac{F(\tau)}{\widetilde{F}_{1}(\tau)},
$$

we have $P\left(t, f_{1}\right)=0$, where $P(x, z)$ is the polynomial in [41]. By [12, 13], we have $t\left(\tau_{0}\right)=-3^{7} / 2^{10}$ and $f_{1}\left(\tau_{0}\right)$ is equal to one of the six zeros of the polynomial $P\left(-3^{7} / 2^{10}, z\right)$ in $z$. To see which zero is equal to $f_{1}\left(\tau_{0}\right)$, we note that $\tau_{0}$, as a point on $X_{6}^{*}(5)$, is one of two CM-points of discriminant -19 and the these two CM-points of discriminant -19 are rational over $\mathbb{Q}(\sqrt{-19})$. Therefore, $f_{1}\left(\tau_{0}\right)$ is equal to one of $512(19 \pm \sqrt{-19}) / 60021$. Now

$$
\frac{d}{d \tau} \log \frac{F(\tau)}{\widetilde{F}_{1}(\tau)}=\frac{F^{\prime}(\tau)}{F(\tau)}-\frac{20}{(c \tau+d+1)^{2}} \frac{F^{\prime}\left(\gamma_{1} \tau\right)}{F\left(\gamma_{1} \tau\right)}+\frac{8 c}{c \tau+d+1} .
$$

Evaluating at $\tau_{0}$, we get

$$
\left.\frac{d}{d \tau} \log \frac{F(\tau)}{\widetilde{F}_{1}(\tau)}\right|_{\tau=\tau_{0}}=\frac{2 \sqrt{-19}}{1+\sqrt{-19}} \frac{F^{\prime}\left(\tau_{0}\right)}{F\left(\tau_{0}\right)}+\frac{8 c}{1+\sqrt{-19}} .
$$

Comparing with (19), we find

$$
\left.\frac{d}{d \tau} \log \frac{F(\tau)}{\widetilde{F}(\tau)}\right|_{\tau=\tau_{0}}=\left.\frac{1+\sqrt{-19}}{\sqrt{-19}} \frac{d}{d \tau} \log \frac{F(\tau)}{\widetilde{F}_{1}(\tau)}\right|_{\tau=\tau_{0}} .
$$

Since $S^{\prime}$ is necessarily real, we conclude that

$$
\left.\frac{d}{d \tau} \log \frac{F(\tau)}{\widetilde{F}_{1}(\tau)}\right|_{\tau=\tau_{0}}=\frac{512(19+\sqrt{-19})}{60021},\left.\quad \frac{d}{d \tau} \log \frac{F(\tau)}{\widetilde{F}(\tau)}\right|_{\tau=\tau_{0}}=\frac{10240}{60021} .
$$


Substituting this into (42), we obtain $S^{\prime}=15309 / 15808$ and (16) and (17) become

$$
\frac{2^{25 / 3} \cdot 13 \cdot 19^{1 / 2}}{3^{28 / 3}} \sum_{n=0}^{\infty}\left(12 \cdot \frac{3^{7}}{2^{10}} n+\frac{15309}{15808}\right) B_{n}\left(-\frac{2^{10}}{3^{7}}\right)^{n}=\frac{4 \sqrt{2}}{\sqrt{19}} C_{2}
$$

and

$\frac{2^{25 / 3} \cdot 13 \cdot 19^{1 / 2}}{3^{28 / 3}} \sum_{n=0}^{\infty}\left(12 \cdot \frac{3^{7}}{2^{10}}(n+1 / 3)+\frac{15309}{15808}\right) B_{n}^{\prime}\left(-\frac{2^{10}}{3^{7}}\right)^{n}=\frac{3^{7 / 3}}{2^{5 / 6} \cdot 19^{1 / 2}} C_{2}^{-1}$.

This proves the identities for the discriminant -19 .

APPENDIX. LIST OF THE AUXILIARY POLYNOMIALS IN THE PROOF OF THEOREM 1

Here we list the polynomials used to evaluate the quantities $S$ and $S^{\prime}$ in Theorem 2 , corresponding to the Hecke operators $T_{5}, T_{7}, T_{11}$, and $T_{13}$. We have also computed the polynomials corresponding to $T_{17}$ and $T_{19}$, but they are too complicated to be displayed here.

$$
\begin{aligned}
& P_{5}(x, z)=27 x^{3}(x-1)^{2}(138240 x+14641) z^{6}+7464960 x^{3}(x-1)^{2} z^{5} \\
&+ 1080 x^{2}(x-1)(5760 x-5041) z^{4}+8640 x(x-1)(320 x-183) z^{3} \\
&+ 2160\left(320 x^{2}-363 x+75\right) z^{2}+18432(5 x-3) z+5120 . \\
& P_{7}(x, z)=2 \\
&+ \\
&+ 135661 x^{6}(x-1)^{3}(3024000000 x-4097152081) z^{8} \\
&+ 31129925400 x^{5}(x-1)^{2}(3600000 x-1210687) z^{6} \\
&+ 1245197016000 x^{4}(x-1)^{2}(40000 x-3471) z^{5} \\
&+ 648270000 x^{3}(x-1)\left(19208000 x^{2}-20441799 x+253587\right) z^{4} \\
&+ 484041600000 x^{3}(x-1)(3430 x-4083) z^{3} \\
&+ 196000000 x^{2}\left(470596 x^{2}-1267231 x+809757\right) z^{2} \\
&-77760000000 x(70 x-79) z+97200000000
\end{aligned}
$$




$$
\begin{aligned}
P_{11}(x, z)= & 3^{3} \cdot 11^{11} x^{9}(x-1)^{5}\left(55427328000000000000 x^{2}\right. \\
& -49446923464224000000 x+16546678259573027281) z^{12} \\
+ & 146426514344294976000000 x^{9}(x-1)^{5}(11664000000 x-5202748681) z^{11} \\
+ & 3081366042598800 x^{8}(x-1)^{4}\left(1016167680000000000 x^{2}\right. \\
& -1049726627175600000 x+115543881567821837) z^{10} \\
+ & 616273208519760000 x^{7}(x-1)^{4}\left(5645376000000000 x^{2}\right. \\
& -3677542804408000 x+127465483643709) z^{9} \\
+ & 115753795740000 x^{6}(x-1)^{3}\left(22541986368000000000 x^{3}\right. \\
& -29303542018872968000 x^{2}+8367736299973548993 x \\
& -48085474493471259) z^{8} \\
+ & 67908893500800000 x^{6}(x-1)^{3}\left(20492714880000000 x^{2}\right. \\
& -20033338665168220 x+3718941297294093) z^{7} \\
+ & 7086244000000 x^{5}(x-1)^{2}\left(76372249814784000000 x^{3}\right. \\
& -128575618679598352328 x^{2}+57998197119448630451 x \\
& -5663679419932760835) z^{6} \\
+ & 7653143520000000 x^{4}(x-1)^{2}\left(20204298892800000 x^{3}\right. \\
& -28634937172769840 x^{2}+9248623134907827 x \\
& -457073405776467) z^{5} \\
+ & 4392300000000 x^{3}(x-1)\left(7334160498086400000 x^{4}\right. \\
& -15976959347457604672 x^{3}+10447013111651394091 x^{2} \\
& -1816427912401084950 x+28962670375592235) z^{4} \\
+ & 556592256000000000 x^{3}(x-1)\left(8574355240000 x^{3}\right. \\
& \left.-16864516118791 x^{2}+9292154719289 x-906852132306\right) z^{3} \\
+ & 87120000000000 x^{2}\left(5477984075731200 x^{4}-15242678431737988 x^{3}\right. \\
& \left.+14384207946457063 x^{2}-4779637835923170 x+160347688352127\right) z^{2} \\
+ & 2^{18} \cdot 3^{2} \cdot 5^{11} x\left(251074270137680 x^{4}-659218490328772 x^{3}\right. \\
+ & \left.581453582393871 x^{2}-172920969584946 x-313911658089\right) z \\
\hline & \cdot 5^{12}\left(50214854027536 x^{4}-125349434637768 x^{3}\right. \\
& \left.+105702451736409 x^{2}-30494063413242 x+3^{6} \cdot 31^{4}\right) . \\
&
\end{aligned}
$$




$$
\begin{aligned}
& 22 \text { YIFAN YANG } \\
& P_{13}(x, z)=220795952705752437 x^{9}(x-1)^{7}\left(4298005400832000000000000 x^{2}\right. \\
& +13214513377973804832000000 x+86160445679273570730609121) z^{14} \\
& +4959960281582022744768000000 x^{9}(x-1)^{7} \\
& (765314352000000 x+1176505820688551) z^{13} \\
& +2296277908139825344800 x^{8}(x-1)^{6}\left(3030644833920000000000 x^{2}\right. \\
& -783773604230560200000 x-5630164854296621557201) z^{12} \\
& +229627790813982534480000 x^{7}(x-1)^{6}\left(33673831488000000000 x^{2}\right. \\
& -17579212510185032000 x-8618813715246332883) z^{11} \\
& +2009976811158420000 x^{6}(x-1)^{5}\left(2885274903386304000000000 x^{3}\right. \\
& -4103184318785684647112000 x^{2}+2044007448506382902634455 x \\
& +30157407046005622302021) z^{10} \\
& +1393583922403171200000 x^{6}(x-1)^{5}\left(2219442233374080000000 x^{2}\right. \\
& -2426222895096718949060 x+787891279616791949667) z^{9} \\
& +88098917868000000 x^{5}(x-1)^{4}\left(13653120842823990528000000 x^{3}\right. \\
& -22388427898915331670097928 x^{2}+9169755921417583303317311 x \\
& -1335510272388880812298095) z^{8} \\
& +2439662340960000000 x^{4}(x-1)^{4}\left(140865532505326886400000 x^{3}\right. \\
& -160884774367727917569920 x^{2}+30658539719491288264803 x \\
& -2202805319008915077183) z^{7} \\
& +169420995900000000 x^{3}(x-1)^{3}\left(422596597515980659200000 x^{4}\right. \\
& -702320292170391172660096 x^{3}+322825169771624225610931 x^{2} \\
& -17212195753867123465230 x+521150155117197321483) z^{6} \\
& +108429437376000000000 x^{3}(x-1)^{3}\left(97823286462032560000 x^{3}\right. \\
& -122470413622723541518 x^{2}+40960825408914015813 x \\
& +487853844789766851) z^{5} \\
& +30845880000000000 x^{2}(x-1)^{2}\left(34386841657133685491200 x^{4}\right. \\
& -67942078041333868279336 x^{3}+39789568931225592998025 x^{2} \\
& -7309981850726385482262 x-213332931298137524427) z^{4} \\
& +1645113600000000000 x(x-1)^{2}\left(39075956428561006240 x^{4}\right. \\
& -77239218331736761300 x^{3}+33148967185029083505 x^{2} \\
& -3997021281851275353 x-65453273808200820) z^{3} \\
& +676000000000000(x-1)\left(2641534654570724021824 x^{5}\right. \\
& -10403175406132029675440 x^{4}+9904647406736445306112 x^{3} \\
& -2232299734677813245211 x^{2}+121697198509392431190 x \\
& -992100687686581707) z^{2} \\
& -622080000000000000(x-1)\left(202316342455567340 x^{3}-249951695360269903 x^{2}\right. \\
& +27606248088252470 x-201317498982675) z \\
& +1555200000000000000\left(1445116731825481 x^{2}-1135066723251890 x\right. \\
& +7456203666025) \text {. }
\end{aligned}
$$




\section{REFERENCES}

[1] Montserrat Alsina and Pilar Bayer. Quaternion orders, quadratic forms, and Shimura curves, volume 22 of CRM Monograph Series. American Mathematical Society, Providence, RI, 2004.

[2] George E. Andrews, Richard Askey, and Ranjan Roy. Special functions, volume 71 of Encyclopedia of Mathematics and its Applications. Cambridge University Press, Cambridge, 1999.

[3] Nayandeep Deka Baruah, Bruce C. Berndt, and Heng Huat Chan. Ramanujan's series for $1 / \pi$ : a survey. Amer. Math. Monthly, 116(7):567-587, 2009.

[4] Bruce C. Berndt and Heng Huat Chan. Eisenstein series and approximations to $\pi$. Illinois J. Math., 45(1):7590, 2001.

[5] Jonathan M. Borwein and Peter B. Borwein. Pi and the AGM. Canadian Mathematical Society Series of Monographs and Advanced Texts. John Wiley \& Sons Inc., New York, 1987. A study in analytic number theory and computational complexity, A Wiley-Interscience Publication.

[6] Jonathan M. Borwein and Peter B. Borwein. Class number three Ramanujan type series for $1 / \pi$. J. Comput. Appl. Math., 46(1-2):281-290, 1993. Computational complex analysis.

[7] Heng Huat Chan and Wen-Chin Liaw. Cubic modular equations and new Ramanujan-type series for $1 / \pi$. Pacific J. Math., 192(2):219-238, 2000.

[8] Heng Huat Chan, Wen-Chin Liaw, and Victor Tan. Ramanujan's class invariant $\lambda_{n}$ and a new class of series for 1/ $\pi$. J. London Math. Soc. (2), 64(1):93-106, 2001.

[9] Heng Huat Chan, Yoshio Tanigawa, Yifan Yang, and Wadim Zudilin. New analogues of Clausen's identities arising from the theory of modular forms. Adv. Math., 228(2):1294-1314, 2011.

[10] Heng Huat Chan and Helena Verrill. The Apéry numbers, the Almkvist-Zudilin numbers and new series for

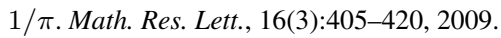

[11] David V. Chudnovsky and Gregory V. Chudnovsky. Approximations and complex multiplication according to Ramanujan. In Ramanujan revisited (Urbana-Champaign, Ill., 1987), pages 375-472. Academic Press, Boston, MA, 1988.

[12] Noam D. Elkies. Shimura curve computations. In Algorithmic number theory (Portland, OR, 1998), volume 1423 of Lecture Notes in Comput. Sci., pages 1-47. Springer, Berlin, 1998.

[13] Eric Errthum. Singular moduli of Shimura curves. Canad. J. Math., 63(4):826-861, 2011.

[14] Srinivasa Ramanujan. Modular equations and approximations to $\pi$. Quart. J. Math. Oxford Ser. (2), 45:350$372,1914$.

[15] Atle Selberg and Sarvadaman Chowla. On Epstein's zeta-function. J. Reine Angew. Math., 227:86-110, 1967.

[16] Marie-France Vignéras. Arithmétique des algèbres de quaternions, volume 800 of Lecture Notes in Mathematics. Springer, Berlin, 1980.

[17] John Voight. Shimura curve computations. In Arithmetic geometry, volume 8 of Clay Math. Proc., pages 103-113. Amer. Math. Soc., Providence, RI, 2009.

[18] Yifan Yang. Schwarzian differential equations and Hecke eigenforms on Shimura curves. Compositio Math., 149:1-31, 2013.

[19] Wadim Zudilin. Ramanujan-type formulae for $1 / \pi$ : a second wind? In Modular forms and string duality, volume 54 of Fields Inst. Commun., pages 179-188. Amer. Math. Soc., Providence, RI, 2008.

Department of Applied Mathematics, National Chiao Tung University and National CenTER FOR THEORETICAL SCIENCES, HSINCHU, TAIWAN 300

E-mail address: yfyang@math.nctu.edu.tw 